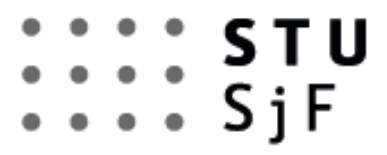

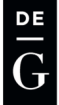

DE GRUYTER OPEN

Print ISSN 0039-2472

On-line ISSN 2450-5471

Journal of MECHANICAL ENGINEERING

- Strojnícky časopis

VOLUME 65, NO 1, 2015

pp. $57-76$

DOI:10.1515/scjme-2016-0004

\title{
EVALUATION OF FRACTURE PARAMETERS FOR CRACKS IN COUPLED THERMOELASTICITY FOR FUNCTIONALLY GRADED MATERIALS
}

\author{
REPKA, M. ${ }^{1}$, SLÁDEK, J. ${ }^{1}$, SLÁDEK, V. ${ }^{1}$, WÜNSCHE, M. ${ }^{2}$ \\ ${ }^{1}$ Institute of Construction and Architecture, Slovak Academy of Sciences, 84503 Bratislava, Slovakia, email: \\ miroslav.repka@savba.sk \\ ${ }^{2}$ Department of Civil Engineering, Germany.
}

\begin{abstract}
The finite element method (FEM) is developed for coupled thermoelastic crack problems if material properties are continuously varying. The weak form is utilized to derive the FEM equations. In conventional fracture theories the state of stress and strain at the crack tip vicinity is characterized by a single fracture parameter, namely the stress intensity factor or its equivalent, J-integral. In the present paper it is considered also the second fracture parameter called as the T-stress. For evaluation of both fracture parameters the quarter-point crack tip element is developed. Simple formulas for both fracture parameters are derived comparing the variation of displacements in the quarter-point element with asymptotic expression of displacement at the crack tip vicinity. The leading terms of the asymptotic expansions of fields in the crack-tip vicinity in a functionally graded material (FGM) are the same as in a homogeneous one with material coefficients taken at the crack tip.
\end{abstract}

KEYWORDS: FGM, thermoelasticity, FEM, fracture, SIFS, T-stresses

\section{Introduction}

Cracking of structures due to a thermal load is very important in many industrial applications. Only composite materials can resist high temperatures effectively. To manufacture a structural component with a constant mixture ratio of ceramics and metals is not efficient way due to specific requirements on individual parts of structural surfaces. A more sophisticated way is, if the volume fractions composite constituents are varied continuously. Functionally graded materials (FGMs) possess continuously nonhomogeneous material properties and they have been introduced in recent years to benefit from the superior performance of its constituents, e.g. high heat and corrosion resistances of ceramics on one side, and large mechanical strength and toughness of metals on the other side. Ceramics are brittle materials and micro-cracks play crucial roles in determining the strength and lifetime of components. A review on the FGMs can be found in the monograph of Suresh and Mortensen (1998) and the review chapter by Paulino et al. (2003). Fracture problems in isotropic FGMs have been investigated by several authors, see e.g., Erdogan (1995); Sladek et al. (2000); Rao and Rahman (2003); Kim and Paulino (2002); Dolbow and Gosz (2002); and Yue et al. (2003). 
In early theories of fracture mechanics assume that the stress and displacement states at the crack tip vicinity are controlled by only one fracture parameter, the stress intensity factor (SIF). The SIFs are necessary for the calculation of the residual strength of the cracked structures, the evaluation of critical crack-lengths and the deduction of rates of the fatigue crack growth. Many efficient techniques for evaluation the SIF in nonhomogeneous solids exist in the literature (Noda and Jin, 1993a,b; Jin and Noda, 1993a,b; Nemat-Alla and Noda, 1996, Gu and Asaro 1997). Ozturk and Erdogan (1997, 1999) used the singular integral equation method to investigate mode-I and mixed-mode crack problems in an infinite nonhomogeneous orthotropic medium with a crack aligned to one of the principal material axes and a constant Poisson's ratio. Kim and Paulino (2003b) have proposed the domain interaction integral method for the evaluation of stress intensity factors in orthotropic functionally graded materials.

Numerical and experimental studies in elastostatics (Sumpter, 1993; Betegon and Hancock, 1991; Sumpter and Hancock, 1991) have been attempted to describe fracture by using two parameters such that additional information could be gained. This second fracture parameter is the leading non-singular terms of the crack tip series expansion (Williams, 1957) and it is called as the T-stress. It represents the stress acting parallel to the crack plane. This second fracture parameter offers better predictions of the crack path direction and the stability (Cotterell and Rice, 1980), and fracture toughness in elastic solids under conditions of low crack-tip stress triaxiality (Williams and Ewing, 1972). The T-stress has significant effects on crack growth direction and stability (Williams and Ewing, 1972, Cotterell and Rice, 1980) as well as to have influence on branched and kinked cracks (Selvarathinam and Goree, 1998). The sign and magnitude of the T-stress can substantially affect the crack tip constraint (Larsson and Carlsson, 1973, Rice, 1974). Less attention is devoted to the evaluation of the Tstress. An overview of past research on T-stress has been published recently by Gupta et al. (2015).

Accurate computational methods for evaluation of T-stress are needed. While this parameter can be computed directly from the asymptotic expansion of stresses or displacements, the results are sensitive to the distance of a selected point from the crack tip. It would be more expedient to obtain the T-stress by an integral formula on a contour far away from the crack tip so to avoid the effect of singular stresses. Many possibilities exist for evaluation of the T-stress (Leevers and Radon, 1982, Kfouri, 1986, Sherry et al., 1995, Nakamura and Parks, 1992, Olsen, 1994, Sladek and Sladek, 1997a,b; Sladek et al., 1997c, Smith et al., 2001, Kim and Paulino, 2003a, Shah et al., 2005, Phan, 2011). However, in all above mentioned papers, the authors have investigated the T-stress only in homogeneous bodies. Only, Kim and Paulino (2004) presented a computational method for the evaluation of the T-stress in orthotropic functionally graded materials. However, their work is devoted only to problems under a static load. The method is based on the interaction integral expressed by a domain integral. It requires an accurate evaluation of quantities occurring in the domain integral at the crack-tip vicinity. It is well known that the accuracy of the computed stress and displacement fields at the crack-tip vicinity is lower than for ones far away from the crack-tip. Therefore, a contour-domain integral formulation for the evaluation of stress intensity factors and T-stresses in orthotropic FGMs under thermal and impact mechanical loads is proposed by Sladek et al. (2006). Only the inertial term and the term with the gradients of material parameters occur in the domain integral. However, the contour-domain integral formulation is convenient for boundary discretization methods, like the boundary element method (BEM). In the FEM, the evaluations of field gradients on element interfaces are not accurate. Therefore, 
the domain integral alternative for evaluation of J-integral is preferred in the FEM analyses. In the FEM conventional quarter-point crack-tip elements are used (Barsoum, 1976). The quarter-point element embodies the square root behaviour of displacements at it is convenient also for evaluation of the SIF. Tan and Wang (2003) have derived a special formula for evaluation of the T-stress in homogeneous bodies. Such a quarter-point element has been developed for the BEM.

The aim of the present paper is give a similar formula for the crack elements in the FEM with functionally graded material properties. New formula for evaluation of the T-stress in cracked bodies with FGM properties is applied for problems described by coupled thermoelasticity (Nowacki, 1986). For sophisticated materials such as high performance composites thermal effects can include heat production due to the strain rate, i.e. the thermoelastic dissipation. Suggested computational methods are applied to numerical examples for a thick-walled cracked tube. On both tube surfaces the different temperatures are prescribed. Numerical results for the stress intensity factor and T-stress in the FGM hollow cylinder are compared with those in corresponding homogeneous material.

\section{The FEM in coupled thermoelasticity}

It is well known that in the coupled thermoelasticity the temperature field is affected by strain rate, i.e. the thermoelastic dissipation is observed. Several computational methods have been proposed over the years to solve uncoupled problems in steady or transient heat conduction states. Few investigations have been done successfully for coupled thermoelasticity. Analyses for coupled thermoelasticity in the FGM are very seldom. Further, an orthotropic and linear elastic solid with continuously varying material properties is considered. The balance of momentum and thermal equations in transient coupled thermoelasticity (Nowacki, 1986) can be written as

$$
\begin{aligned}
& \sigma_{i j, j}(\mathbf{x}, \tau)-\rho \ddot{u}_{i}(\mathbf{x}, \tau)+X_{i}(\mathbf{x}, \tau)=0, \\
& {\left[k_{i j}(\mathbf{x}) \theta_{, j}(\mathbf{x}, \tau)\right]_{, i}-\rho c \dot{\theta}(\mathbf{x}, \tau)-\gamma_{i j} \theta_{0} \dot{u}_{i, j}(\mathbf{x}, \tau)+Q(\mathbf{x}, \tau)=0,}
\end{aligned}
$$

where $\sigma_{i j}, \tau, \theta, \theta_{0}, u_{i}, X_{i}$ and $\mathrm{Q}$ are the stress, time, temperature difference, reference temperature, displacement, density of body force vector and density of heat sources, respectively. Also, $\rho, k_{i j}, c$ and $\gamma_{i j}$ are the mass density, thermal conductivity tensor, specific heat, stress-temperature modulus, respectively. The dots over a quantity indicate the time derivatives. A static problem can be considered formally as a special case of the dynamic

one, by omitting the acceleration $\ddot{u}_{i}(\mathbf{x}, \tau)$ in the equations of motion (1) and the time derivative terms in equation (2). Therefore, both cases are analyzed in this paper simultaneously.

The relation between the stress $\sigma_{i j}$ and the strain $\varepsilon_{i j}$ in thermoelasticity is given by the well known Duhamel-Neumann constitutive equations

$$
\sigma_{i j}(\mathbf{x}, \tau)=c_{i j k l} \varepsilon_{k l}(\mathbf{x}, \tau)-\gamma_{i j} \theta(\mathbf{x}, \tau)
$$


where $c_{i j k l}$ are the material stiffness coefficients. The stress-temperature modulus can be expressed through the stiffness coefficients and the coefficients of linear thermal expansion $\alpha_{k l}$ as

$$
\gamma_{i j}=c_{i j k l} \alpha_{k l}
$$

The constitutive equation (3) can be written in matrix form with introducing the vectors of stress and strain tensor components (Lekhnitskii, 1963). The constitutive equation for orthotropic materials and plane strain problems has the following form

$$
\begin{aligned}
& {\left[\begin{array}{l}
\sigma_{11} \\
\sigma_{22} \\
\sigma_{12}
\end{array}\right]=\left[\begin{array}{ccc}
c_{11} & c_{12} & 0 \\
c_{12} & c_{22} & 0 \\
0 & 0 & c_{66}
\end{array}\right]\left[\begin{array}{l}
\varepsilon_{11} \\
\varepsilon_{22} \\
2 \varepsilon_{12}
\end{array}\right]-\left[\begin{array}{ccc}
c_{11} & c_{12} & c_{13} \\
c_{12} & c_{22} & c_{23} \\
0 & 0 & 0
\end{array}\right]\left[\begin{array}{l}
\alpha_{11} \\
\alpha_{22} \\
\alpha_{33}
\end{array}\right] \theta=\mathbf{C}\left[\begin{array}{c}
\varepsilon_{11} \\
\varepsilon_{22} \\
2 \varepsilon_{12}
\end{array}\right]-\gamma \theta,} \\
& \text { with } \boldsymbol{\gamma}=\left[\begin{array}{ccc}
c_{11} & c_{12} & c_{13} \\
c_{12} & c_{22} & c_{23} \\
0 & 0 & 0
\end{array}\right]\left[\begin{array}{l}
\alpha_{11} \\
\alpha_{22} \\
\alpha_{33}
\end{array}\right]=\left[\begin{array}{c}
\gamma_{11} \\
\gamma_{22} \\
0
\end{array}\right] .
\end{aligned}
$$

Equation (5) can be reduced to a simple form for isotropic materials

$$
\sigma_{i j}=2 \mu \varepsilon_{i j}+\lambda \varepsilon_{k k} \delta_{i j}-(3 \lambda+2 \mu) \alpha \theta \delta_{i j},
$$

with Lame's constants $\lambda, \mu$, and the coefficient of linear thermal expansion $\alpha$.

For a plane stress state of a 2-d anisotropic elastic body, the generalized Hooke's law can be written [Lekhnitskii (1963)] as

$$
\left[\begin{array}{c}
\varepsilon_{11} \\
\varepsilon_{22} \\
2 \varepsilon_{12}
\end{array}\right]=\left[\begin{array}{lll}
a_{11} & a_{12} & a_{16} \\
a_{12} & a_{22} & a_{26} \\
a_{16} & a_{26} & a_{66}
\end{array}\right]\left[\begin{array}{l}
\sigma_{11} \\
\sigma_{22} \\
\sigma_{12}
\end{array}\right] \text {, }
$$

where $a_{i j}$ are the elastic compliances of the material. In the case of plane strain conditions, the coefficients $a_{i j}$ should be replaced by $\tilde{a}_{i j}$, where

$$
\tilde{a}_{i j}=a_{i j}-\frac{a_{i 3} a_{j 3}}{a_{33}}
$$

The compliance coefficients can be expressed in terms of engineering constants as

$$
\begin{array}{ll}
a_{11}=1 / E_{1}, \quad a_{22}=1 / E_{2}, & a_{33}=1 / E_{3} \\
a_{12}=-v_{12} / E_{1}=-v_{21} / E_{2}, & a_{16}=\eta_{12,1} / E_{1}=\eta_{1,12} / \mu \\
a_{26}=\eta_{12,2} / E_{2}=\eta_{2,12} / \mu, & a_{66}=1 / \mu,
\end{array}
$$


where $E_{k}$ are the Young's moduli refering to the axes $x_{k}, \mu_{\text {is }}$ the shear modulus for the plane, $v_{i j}$ are Poisson's ratios and $\eta_{j k, l}$ and $\eta_{l, j k}$ are the mutual coefficients of first and second kind, respectively. For orthotropic materials $a_{16}=a_{26}=0$.

The following essential and natural boundary conditions are assumed for the mechanical quantities

$$
\begin{aligned}
& u_{i}(\mathbf{x}, \tau)=\tilde{u}_{i}(\mathbf{x}, \tau) \quad \text { on } \quad \Gamma_{u}, \\
& t_{i}(\mathbf{x}, \tau)=\sigma_{i j}(\mathbf{x}, \tau) n_{j}(\mathbf{x})=\tilde{t}_{i}(\mathbf{x}, \tau) \quad \text { on } \quad \Gamma_{t},
\end{aligned}
$$

and for the thermal quantities

$$
\begin{aligned}
& \theta(\mathbf{x}, \tau)=\tilde{\theta}(\mathbf{x}, \tau) \quad \text { on } \quad \Gamma_{p}, \\
& q(\mathbf{x}, \tau)=-k_{i j} \theta_{, j}(\mathbf{x}, \tau) n_{i}(\mathbf{x})=\tilde{q}(\mathbf{x}, \tau) \quad \text { on } \quad \Gamma_{q},
\end{aligned}
$$

where $\Gamma_{u}$ is the part of the global boundary with prescribed displacements, while on $\Gamma_{t}$, $\Gamma_{p}$ and $\Gamma_{q}$ the traction vector $t_{i}$, temperature and the heat flux $q$ are prescribed, respectively.

Initial conditions for the mechanical and thermal quantities have to be prescribed

$$
\begin{aligned}
& \left.u_{i}(\mathbf{x}, \tau)\right|_{\tau=0}=u_{i}(\mathbf{x}) \text { and }\left.\dot{u}_{i}(\mathbf{x}, \tau)\right|_{\tau=0}=\dot{u}_{i}(\mathbf{x}) \\
& \left.\theta(\mathbf{x}, \tau)\right|_{\tau=0}=\theta(\mathbf{x}) \text { in } \Omega .
\end{aligned}
$$

The weak form of the governing equation (1) is given as:

$$
\int_{\Omega}\left[\sigma_{i j, j}(\mathbf{x}, \tau)-\rho \ddot{u}_{i}(\mathbf{x}, \tau)+X_{i}(\mathbf{x}, \tau)\right] w_{i} d \Omega=0,
$$

where $w_{i}$ is a test function. The test function is selected such a way that it is vanishing on $\Gamma_{u}$ where Dirichlet boundary conditions are considered. Applying Gauss-Green's theorem to the first domain integral, one can write:

$$
-\int_{\Omega} \sigma_{i j}(\mathbf{x}, \tau) w_{i, j} d \Omega+\int_{\Gamma_{t}} \tilde{t}_{i}(\mathbf{x}, \tau) w_{i} d \Gamma+\int_{\Omega}\left[-\rho \ddot{u}_{i}(\mathbf{x}, \tau)+X_{i}(\mathbf{x}, \tau)\right] w_{i} d \Omega=0 .
$$

Similarly one can rewrite the governing equation (2) into the weak form

$$
\int_{\Omega}\left\{\left[k_{i j}(\mathbf{x}) \theta_{, j}(\mathbf{x}, \tau)\right]_{, i}-\rho c \dot{\theta}(\mathbf{x}, \tau)-\gamma_{i j} \theta_{0} \dot{u}_{i, j}(\mathbf{x}, \tau)+Q(\mathbf{x}, \tau)\right\} \theta^{*} d V=0
$$


where $\theta^{*}$ is the test function for the second governing equation. Applying Gauss-Green's theorem to the weak form (14), one can write

$$
\begin{aligned}
& -\int_{\Omega} k_{i j}(\mathbf{x}) \theta_{, j}(\mathbf{x}, \tau) \theta_{, i}^{*} d \Omega-\int_{\Gamma_{q}} \tilde{q}(\mathbf{x}, \tau) \theta^{*} d \Gamma+ \\
& \quad+\int_{\Omega}\left[-\rho c \dot{\theta}(\mathbf{x}, \tau)-\gamma_{i j} \theta_{0} \dot{u}_{i, j}(\mathbf{x}, \tau)+Q(\mathbf{x}, \tau)\right] \theta^{*} d \Omega=0 .
\end{aligned}
$$

Now we define auxiliary fields for test functions:

$$
W_{i j}=\frac{1}{2}\left(w_{i, j}+w_{j, i}\right) \quad \text { and } \quad O_{i}=\theta_{, i}^{*}
$$

Then, one can write $\sigma_{i j} w_{i, j}=\sigma_{j i} w_{i, j}=\sigma_{j i} w_{j, i}=\frac{1}{2} \sigma_{i j}\left(w_{i, j}+w_{j, i}\right)=\sigma_{i j} W_{i j}$.

We can also define matrices for stress, strain, displacement, body force vector in $2 \mathrm{D}$ and traction vector as:

$$
\begin{aligned}
\tilde{\boldsymbol{\sigma}} & =\left[\begin{array}{lll}
\sigma_{11} & \sigma_{22} & \sigma_{12}
\end{array}\right]^{T}, \\
\tilde{\boldsymbol{\varepsilon}} & =\left[\begin{array}{lll}
\varepsilon_{11} & \varepsilon_{22} & 2 \varepsilon_{12}
\end{array}\right]^{T} ; \\
\tilde{\mathbf{u}} & =\left[\begin{array}{ll}
u_{1} & u_{2}
\end{array}\right]^{T} ; \quad \mathbf{X}=\left[\begin{array}{ll}
X_{1} & X_{2}
\end{array}\right]^{T} ; \quad \tilde{\mathbf{t}}=\left[\begin{array}{ll}
\tilde{t}_{1} & \tilde{t}_{2}
\end{array}\right]^{T}
\end{aligned}
$$

and we can define similar vectors associated with the test functions:

$$
\begin{aligned}
& \mathbf{W}_{\varepsilon}=\left[\begin{array}{lll}
W_{11} & W_{22} & 2 W_{12}
\end{array}\right]^{T} ; \\
& \mathbf{w}=\left[\begin{array}{ll}
w_{1} & w_{2}
\end{array}\right]^{T}
\end{aligned}
$$

Now, the equation (13) can be rewritten into the matrix form:

$$
\int_{\Omega} \mathbf{W}_{\varepsilon}^{T} \tilde{\boldsymbol{\sigma}} d \Omega+\int_{\Omega} \rho \mathbf{w}^{T} \ddot{\mathbf{u}} d \Omega=\int_{\Gamma_{t}} \mathbf{w}^{T} \tilde{\mathbf{t}} d \Gamma+\int_{\Omega} \mathbf{w}^{T} \mathbf{X} d \Omega
$$

Note also that eq. (3) can be arranged in matrix form as:

$$
\tilde{\boldsymbol{\sigma}}=\mathbf{C} \tilde{\boldsymbol{\varepsilon}}-\gamma \theta,
$$

Substituting eq. (20) into (19), we get:

$$
\int_{\Omega} \mathbf{W}_{\boldsymbol{\varepsilon}}^{T} \mathbf{C} \tilde{\boldsymbol{\varepsilon}} d \Omega-\int_{\Omega} \mathbf{W}_{\varepsilon}^{T} \gamma \theta d \Omega+\int_{\Omega} \rho \mathbf{w}^{T} \ddot{\mathbf{u}} d \Omega=\int_{\Gamma_{t}} \mathbf{w}^{T} \tilde{\mathbf{t}} d \Gamma+\int_{\Omega} \mathbf{w}^{T} \mathbf{X} d \Omega
$$

Similarly one can rewrite equation (15) into the matrix form 


$$
\int_{\Omega} \mathbf{O}^{T} \mathbf{q} d \Omega+\int_{\Omega} \rho c \theta^{*} \dot{\theta} d \Omega+\theta_{0} \int_{\Omega} \gamma^{T} \dot{\tilde{\mathbf{\varepsilon}}} d \Omega=\int_{\Gamma_{q}} \theta^{*} \tilde{q} d \Gamma+\int_{\Omega} \theta^{*} Q d \Omega,
$$

where

$$
\mathbf{O}=\left[\begin{array}{l}
\theta_{, 1}^{*} \\
\theta_{, 2}^{*}
\end{array}\right], \quad \mathbf{q}=\left[\begin{array}{l}
q_{1} \\
q_{2}
\end{array}\right]=-\mathbf{k}\left[\begin{array}{l}
\theta_{, 1} \\
\theta_{, 2}
\end{array}\right], \quad \mathbf{k}=\left[\begin{array}{ll}
k_{11} & k_{12} \\
k_{21} & k_{22}
\end{array}\right],
$$

In the finite elements method, we express the primal variables $\theta$ and $\tilde{\mathbf{u}}$ in terms of the shape functions and nodal variables in each element:

$$
\begin{aligned}
& \theta=\mathbf{N} \Theta, \\
& \tilde{\mathbf{u}}=\mathbf{M U}=\left[\begin{array}{ll}
\mathbf{N} & \mathbf{0} \\
\mathbf{0} & \mathbf{N}
\end{array}\right] \mathbf{U},
\end{aligned}
$$

where $\mathbf{N}=\left[\begin{array}{lll}N^{(1)} & N^{(2)} & \cdots\end{array}\right]$ is the shape functions matrix and $\mathbf{U}, \boldsymbol{\Theta}$ are the nodal degrees of freedom vectors in $2 \mathrm{D}: \quad \mathbf{U}=\left[\begin{array}{ll}\mathbf{U}_{1} & \mathbf{U}_{2}\end{array}\right]^{T}, \quad \mathbf{U}_{k}=\left[\begin{array}{lll}u_{k}^{(1)} & u_{k}^{(2)} & \ldots\end{array}\right]^{T}$, $\boldsymbol{\Theta}=\left[\begin{array}{lll}\theta^{(1)} & \theta^{(2)} & \ldots\end{array}\right]^{T}$

where the superscript $(a)$ indicates the node number. The number of shape functions and their expressions depend on the selected element shape and order. We can express the strain tensor as well as the gradients of the temperature in terms of nodal degrees freedom as:

$$
\tilde{\boldsymbol{\varepsilon}}=\left[\begin{array}{cc}
\partial_{1} & 0 \\
0 & \partial_{2} \\
\partial_{2} & \partial_{1}
\end{array}\right] \tilde{\mathbf{u}}=\mathbf{B U}, \quad \mathbf{q}=-\mathbf{k}\left[\begin{array}{l}
\partial_{1} \\
\partial_{2}
\end{array}\right] \theta=-\mathbf{k} \tilde{\mathbf{B}} \boldsymbol{\Theta}
$$

where the matrices $\mathbf{B}$ and $\tilde{\mathbf{B}}$ are expressed in terms of the derivatives of the shape functions and the Jacobian of the transformation from the global Cartesian coordinates to the local coordinates within the finite elements, since the shape functions are defined in the intrinsic coordinate space $\left(\xi_{1}, \xi_{2}\right)$. The Jacobian of the used transformation is defined as

$$
\mathbf{J}=\left[\begin{array}{ll}
\frac{\partial x_{1}}{\partial \xi_{1}} & \frac{\partial x_{1}}{\partial \xi_{2}} \\
\frac{\partial x_{2}}{\partial \xi_{1}} & \frac{\partial x_{2}}{\partial \xi_{2}}
\end{array}\right]=\left[\begin{array}{ccc}
x_{1}^{(1)} & x_{1}^{(2)} & \ldots \\
x_{2}^{(1)} & x_{2}^{(2)} & \ldots
\end{array}\right]\left[\begin{array}{cc}
N_{, 1}^{(1)} & N_{, 2}^{(1)} \\
N_{, 1}^{(2)} & N_{, 2}^{(2)} \\
\vdots & \vdots
\end{array}\right], \quad N_{, i}^{(k)}=\frac{\partial N^{(k)}}{\partial \xi_{i}}
$$

and the derivatives w.r.t. the Cartesian coordinates are expressed in terms of the derivatives w.r.t. the intrinsic coordinates as 
$\left\{\begin{array}{l}\partial / \partial x_{1} \\ \partial / \partial x_{2}\end{array}\right\}=\mathbf{Y}^{T}\left\{\begin{array}{l}\partial / \partial \xi_{1} \\ \partial / \partial \xi_{2}\end{array}\right\}=\left\{\begin{array}{l}Y_{11} \partial / \partial \xi_{1}+Y_{21} \partial / \partial \xi_{2} \\ Y_{12} \partial / \partial \xi_{1}+Y_{22} \partial / \partial \xi_{2}\end{array}\right\}=\left\{\begin{array}{l}Y_{i 1} \partial / \partial \xi_{i} \\ Y_{i 2} \partial / \partial \xi_{i}\end{array}\right\}, \quad \mathbf{Y}=\mathbf{J}^{-1}$

Thus from (24), we have

$$
\mathbf{B}=\left[\begin{array}{cc}
Y_{i 1} \mathbf{N}_{, i} & \mathbf{0} \\
\mathbf{0} & Y_{i 2} \mathbf{N}_{, i} \\
Y_{i 2} \mathbf{N}_{, i} & Y_{i 1} \mathbf{N}_{, i}
\end{array}\right], \quad \tilde{\mathbf{B}}=\left[\begin{array}{c}
Y_{i 1} \mathbf{N}_{, i} \\
Y_{i 2} \mathbf{N}_{, i}
\end{array}\right], \quad \mathbf{N}_{, i}=\left[\begin{array}{lll}
N_{, i}^{(1)} & N_{, i}^{(2)} & \ldots
\end{array}\right] .
$$

Following Galerkin's method by assuming the test functions $\mathbf{w}$ in the same form as the shape functions, we have:

$$
\mathbf{w}=\mathbf{M}, \quad \mathbf{W}_{\varepsilon}=\mathbf{B},
$$

Substituting eqs. (23), (24) and (25) into (21) gives the finite element equations for an element:

$$
\left(\int_{\Omega} \rho \mathbf{M}^{T} \mathbf{M} d \Omega\right) \ddot{\mathbf{U}}+\left(\int_{\Omega} \mathbf{B}^{T} \mathbf{C B} d \Omega\right) \mathbf{U}+\left(\int_{\Omega} \mathbf{B}^{T} \gamma \mathbf{N} d \Omega\right) \boldsymbol{\Theta}=\int_{\Gamma_{t}} \mathbf{M}^{T} \tilde{\mathbf{t}} d \Gamma+\int_{\Omega} \mathbf{M}^{T} \mathbf{X} d \Omega .
$$

Similarly, in equation (22) we consider following test function

$$
\theta^{*}=\mathbf{N} \quad \text { and } \quad \mathbf{O}=\tilde{\mathbf{B}} .
$$

Then, we get

$$
\begin{gathered}
\left(-\int_{\Omega} \tilde{\mathbf{B}}^{T} \mathbf{k} \tilde{\mathbf{B}} d \Omega\right) \boldsymbol{\Theta}+\left(\int_{\Omega} \rho c \mathbf{N}^{T} \mathbf{N} d \Omega\right) \dot{\boldsymbol{\Theta}}+\theta_{0}\left(\int_{\Omega} \gamma^{T} \mathbf{B} d \Omega\right) \dot{\mathbf{U}}= \\
=\int_{\Gamma_{q}} \mathbf{N}^{T} \tilde{q} d \Gamma+\int_{\Omega} \mathbf{N}^{T} Q d \Omega
\end{gathered}
$$

These equations can be written in a compact form as:

$$
\begin{gathered}
\mathbf{M}_{u} \ddot{\mathbf{U}}+\mathbf{K}_{u u} \mathbf{U}+\mathbf{K}_{u \theta} \boldsymbol{\Theta}=\mathbf{R}_{u}, \\
\mathbf{M}_{\theta} \dot{\boldsymbol{\Theta}}+\mathbf{K}_{\theta \theta} \boldsymbol{\Theta}+\mathbf{K}_{\theta u} \dot{\mathbf{U}}=\mathbf{R}_{\theta},
\end{gathered}
$$

where

$$
\mathbf{K}_{u u}=\int_{\Omega} \mathbf{B}^{T} \mathbf{C B} d \Omega, \quad \mathbf{K}_{u \theta}=\int_{\Omega} \mathbf{B}^{T} \gamma \mathbf{N} d \Omega, \quad \mathbf{M}_{u}=\int_{\Omega} \rho \mathbf{M}^{T} \mathbf{M} d \Omega,
$$




$$
\begin{aligned}
& \mathbf{M}_{\theta}=\int_{\Omega} \rho c \mathbf{N}^{T} \mathbf{N} d \Omega, \\
& \mathbf{K}_{\theta \theta}=-\int_{\Omega} \tilde{\mathbf{B}}^{T} \mathbf{k} \tilde{\mathbf{B}} d \Omega, \\
& \mathbf{R}_{u}=\int_{\Gamma_{t}} \mathbf{M}^{T} \tilde{\mathbf{t}} d \Gamma+\int_{\Omega} \mathbf{M}^{T} \mathbf{X} d \Omega \theta_{\Omega} \boldsymbol{\gamma}^{T} \mathbf{B} d \Omega, \\
& \mathbf{R}_{\theta}=\int_{\Gamma_{q}} \mathbf{N}^{T} \tilde{q} d \Gamma+\int_{\Omega} \mathbf{N}^{T} Q d \Omega .
\end{aligned}
$$

\section{Quarter-point crack-tip element and evaluation of the SIF and T-stress}

The stresses and the displacements at the crack-tip vicinity in a material with a continuous non-homogeneity have the same singularity and angular distributions as that in a homogeneous material (Eischen, 1987). The displacements and the stresses close to the cracktip can be written in an asymptotic form as (Sih, 1965, Shah et al., 2006)

$$
\begin{aligned}
& \sigma_{11}=\frac{1}{\sqrt{2 \pi r}}\left\{K_{I} \operatorname{Re}\left[\frac{\mu_{1}^{t i p} \mu_{2}^{t i p}}{\mu_{1}^{t i p}-\mu_{2}^{t i p}}\left(\frac{\mu_{2}^{t i p}}{\sqrt{\cos \varphi+\mu_{2}^{t i p} \sin \varphi}}-\frac{\mu_{1}^{t i p}}{\sqrt{\cos \varphi+\mu_{1}^{t i p} \sin \varphi}}\right)\right]+\right. \\
& \left.+K_{I I} \operatorname{Re}\left[\frac{1}{\mu_{1}^{t i p}-\mu_{2}^{t i p}}\left(\frac{\left(\mu_{2}^{t i p}\right)^{2}}{\sqrt{\cos \varphi+\mu_{2}^{t i p} \sin \varphi}}-\frac{\left(\mu_{1}^{t i p}\right)^{2}}{\sqrt{\cos \varphi+\mu_{1}^{t i p} \sin \varphi}}\right)\right]\right\}+T \delta_{i 1} \delta_{j 1} \\
& \sigma_{22}=\frac{1}{\sqrt{2 \pi r}}\left\{K_{I} \operatorname{Re}\left[\frac{1}{\mu_{1}^{t i p}-\mu_{2}^{t i p}}\left(\frac{\mu_{1}^{t i p}}{\sqrt{\cos \varphi+\mu_{2}^{t i p} \sin \varphi}}-\frac{\mu_{2}^{t i p}}{\sqrt{\cos \varphi+\mu_{1}^{t i p} \sin \varphi}}\right)\right]+\right. \\
& \left.+K_{I I} \operatorname{Re}\left[\frac{1}{\mu_{1}^{t i p}-\mu_{2}^{t i p}}\left(\frac{1}{\sqrt{\cos \varphi+\mu_{2}^{t i p} \sin \varphi}}-\frac{1}{\sqrt{\cos \varphi+\mu_{1}^{t i p} \sin \varphi}}\right)\right]\right\}, \\
& \sigma_{12}=\frac{1}{\sqrt{2 \pi r}}\left\{K_{I} \operatorname{Re}\left[\frac{\mu_{1}^{t i p} \mu_{2}^{t i p}}{\mu_{1}^{t i p}-\mu_{2}^{t i p}}\left(\frac{1}{\sqrt{\cos \varphi+\mu_{2}^{t i p} \sin \varphi}}-\frac{1}{\sqrt{\cos \varphi+\mu_{1}^{t i p} \sin \varphi}}\right)\right]+\right. \\
& \left.+K_{I I} \operatorname{Re}\left[\frac{1}{\mu_{1}^{t i p}-\mu_{2}^{t i p}}\left(\frac{\mu_{1}^{t i p}}{\sqrt{\cos \varphi+\mu_{2}^{t i p} \sin \varphi}}-\frac{\mu_{1}^{t i p}}{\sqrt{\cos \varphi+\mu_{1}^{t i p} \sin \varphi}}\right)\right]\right\}, \\
& u_{1}=\sqrt{\frac{2 r}{\pi}}\left\{K_{I} \operatorname{Re}\left[\frac{1}{\mu_{1}^{t i p}-\mu_{2}^{t i p}}\left(\mu_{1}^{t i p} p_{2} \sqrt{\cos \varphi+\mu_{2}^{t i p} \sin \varphi}-\mu_{2}^{t i p} p_{1} \sqrt{\cos \varphi+\mu_{1}^{t i p} \sin \varphi}\right)\right]+\right.
\end{aligned}
$$




$$
\begin{aligned}
& \left.+K_{I I} \operatorname{Re}\left[\frac{1}{\mu_{1}^{t i p}-\mu_{2}^{t i p}}\left(p_{2} \sqrt{\cos \varphi+\mu_{2}^{t i p} \sin \varphi}-p_{1} \sqrt{\cos \varphi+\mu_{1}^{t i p} \sin \varphi}\right)\right]\right\}+a_{11} \operatorname{Tr} \cos \varphi \\
& u_{2}=\sqrt{\frac{2 r}{\pi}}\left\{K_{I} \operatorname{Re}\left[\frac{1}{\mu_{1}^{t i p}-\mu_{2}^{t i p}}\left(\mu_{1}^{t i p} q_{2} \sqrt{\cos \varphi+\mu_{2}^{t i p} \sin \varphi}-\mu_{2}^{t i p} q_{1} \sqrt{\cos \varphi+\mu_{1}^{t i p} \sin \varphi}\right)\right]+\right. \\
& \left.+K_{I I} \operatorname{Re}\left[\frac{1}{\mu_{1}^{t i p}-\mu_{2}^{t i p}}\left(q_{2} \sqrt{\cos \varphi+\mu_{2}^{t i p} \sin \varphi}-q_{1} \sqrt{\cos \varphi+\mu_{1}^{t i p} \sin \varphi}\right)\right]\right\}+a_{12} \operatorname{Tr} \sin \varphi
\end{aligned}
$$

where Re denotes the real part of a complex function, $\mu_{i}^{\text {tip }}$ are material parameters at the crack-tip, which are roots of the following characteristic equation [Lekhnitskii (1963)]

$$
a_{11} \mu^{4}-2 a_{16} \mu^{3}+\left(2 a_{12}+a_{66}\right) \mu^{2}-2 a_{26} \mu+a_{22}=0
$$

and

$$
\begin{aligned}
& p_{\alpha}=a_{11} \mu_{\alpha}^{2}-a_{16} \mu_{\alpha}+a_{12} \\
& q_{\alpha}=\left(a_{12} \mu_{\alpha}^{2}-a_{26} \mu_{\alpha}+a_{22}\right) / \mu_{\alpha} .
\end{aligned}
$$

In above equation, polar coordinate system with the origin at the crack-tip is used and the $1^{\text {st }}$ Cartesian coordinate axis lies on the tangent to the crack surface at the crack tip.

The T-stress can be computed directly from the above-mentioned asymptotic expansion of stresses or displacements, if the stress intensity factors and the stress or the displacement values at nodal points close to the crack-tip are obtained from a numerical analysis. In the literature, such a method, where T-stress is computed from the known stress component $\sigma_{11}$, is called the boundary layer method (Sherry et al., 1995). A drawback of this method is the sensitivity of the results with respect to the distance of the evaluation point from the crack-tip. Phan (2011) suggested a non-singular boundary integral formula for determining the T-stress as

$$
T=\lim _{r \rightarrow 0}\left[\sigma_{22}(r, 0)-\sigma_{11}(r, 0)\right] .
$$

Integral representations for individual stresses in (38) contain hypersingular integrals, however, the integral representation for subtract of stresses is non-singular. Therefore, the computational method is appropriate for the BEM.

In the FEM the quarter-point crack-tip elements are very favourite for evaluation of the SIFs. Consider isoparametric element with quadratic approximation in Fig. 1. 


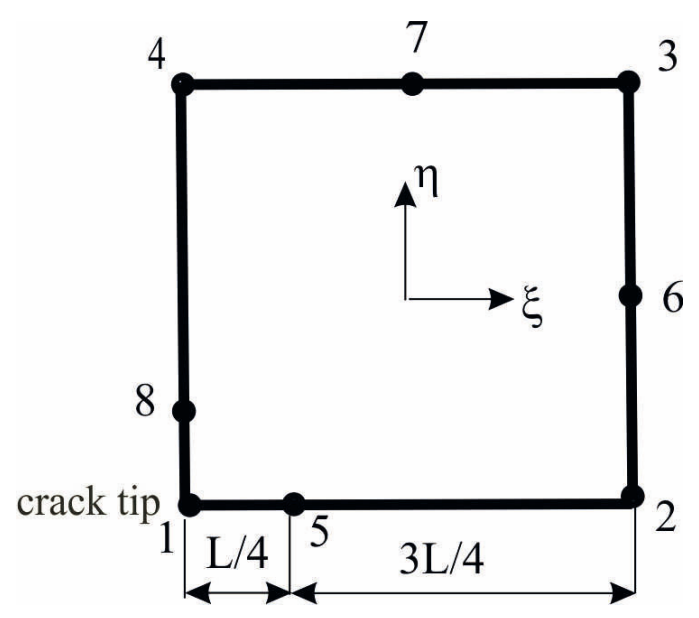

Fig. 1 Quarter-point crack-tip element

The order of singularity for quarter-point element can be found along the line 1-2 in Fig. 1. The shape functions along that line are given by

$$
N^{(1)}=-\frac{1}{2} \xi(1-\xi), \quad N^{(2)}=\frac{1}{2} \xi(1+\xi), \quad N^{(5)}=\left(1-\xi^{2}\right) .
$$

Isoparametric coordinates $(\xi, \eta)$ are from the interval $(-1 \geq \xi \geq 1,-1 \geq \eta \geq 1)$. Any point with coordinate $x$ on line 1-2 can be expressed by nodal coordinates and shape functions

$$
x=-\frac{1}{2} \xi(1-\xi) x^{(1)}+\frac{1}{2} \xi(1+\xi) x^{(2)}+\left(1-\xi^{2}\right) x^{(5)} .
$$

Choosing $x^{(1)}=0, x^{(2)}=L, x^{(5)}=L / 4$, one gets

$$
x=\frac{1}{2} \xi(1+\xi) L+\left(1-\xi^{2}\right) \frac{L}{4} .
$$

Solving the quadratic equation (41) we obtain

$$
\xi=-1+2 \sqrt{\frac{x}{L}}
$$

Similarly, we can approximate the displacement along the line 1-2:

$$
\mathbf{u}=-\frac{1}{2} \xi(1-\xi) \mathbf{u}^{(1)}+\frac{1}{2} \xi(1+\xi) \mathbf{u}^{(2)}+\left(1-\xi^{2}\right) \mathbf{u}^{(5)} \text {. }
$$

Substituting (42) into (43) we get approximation of displacement in quarter-point element

$$
\mathbf{u}=\mathbf{u}^{(1)}+\left(-3 \mathbf{u}^{(1)}+4 \mathbf{u}^{(5)}-\mathbf{u}^{(2)}\right) \sqrt{\frac{x}{L}}+2\left(\mathbf{u}^{(1)}-2 \mathbf{u}^{(5)}+\mathbf{u}^{(2)}\right) \frac{x}{L} .
$$


A similar approximation of displacements can be performed on the opposite crack face. Then, for displacement discontinuities on the crack faces one can write

$$
\Delta \mathbf{u}=\left(4 \Delta \mathbf{u}^{(5)}-\Delta \mathbf{u}^{(2)}\right) \sqrt{\frac{x}{L}}+2\left(-2 \Delta \mathbf{u}^{(5)}+\Delta \mathbf{u}^{(2)}\right) \frac{x}{L},
$$

where $\Delta \mathbf{u}=\mathbf{u}^{+}-\mathbf{u}^{-}$and displacement discontinuities at the crack tip (node 1) has to be vanishing.

Taking into account the asymptotic fields (34) and (35) for $\varphi=\pi$ and equating the coefficients of $\sqrt{r}=\sqrt{x}$ to those in the quarter point element approximation for both components of displacement discontinuities, one can write

$$
\left\{\begin{array}{l}
K_{I} \\
K_{I I}
\end{array}\right\}=\frac{\sqrt{2 \pi}}{4 D \sqrt{L}}\left[\begin{array}{ll}
H_{11} & H_{12} \\
H_{21} & H_{22}
\end{array}\right]\left\{\begin{array}{l}
4 \Delta u^{(5)}-\Delta u^{(2)} \\
4 \Delta v^{(5)}-\Delta v^{(2)}
\end{array}\right\},
$$

where $D=H_{11} H_{22}-H_{12} H_{21}$

$$
\left[\begin{array}{ll}
H_{11} & H_{12} \\
H_{21} & H_{22}
\end{array}\right]=\left[\begin{array}{cc}
\operatorname{Im}\left(\frac{q_{1}-q_{2}}{\mu_{1}-\mu_{2}}\right) & \operatorname{Im}\left(\frac{p_{1}-p_{2}}{\mu_{1}-\mu_{2}}\right) \\
\operatorname{Im}\left(\frac{\mu_{1} q_{1}-\mu_{2} q_{2}}{\mu_{1}-\mu_{2}}\right) & \operatorname{Im}\left(\frac{\mu_{2} q_{1}-\mu_{1} q_{2}}{\mu_{1}-\mu_{2}}\right)
\end{array}\right] .
$$

The stress singularity along the line $1-2$ is proportional $\sqrt{L / x}$. The variation of the traction vector becomes

$$
\mathbf{t}=\left(-3 \mathbf{t}^{(1)}+4 \mathbf{t}^{(5)}-\mathbf{t}^{(2)}\right) \sqrt{\frac{L}{x}}+2\left(\mathbf{t}^{(1)}-2 \mathbf{t}^{(5)}+\mathbf{t}^{(2)}\right) \sqrt{\frac{x}{L}} .
$$

Comparing the coefficients standing at $x=r$ in (44) for displacements and those in (34) with $\varphi=\pi$, we get a simple formula for evaluation of the T-stress

$$
\begin{aligned}
& T=-\frac{2}{a_{11} L}\left[u_{1}^{(1)}-2 u_{1}^{(5)}+u_{1}^{(2)}\right], \\
& \text { where } a_{11}=\left\{\begin{array}{ll}
\frac{1}{E_{1}} & \text { plane stress } \\
\frac{1}{E_{1}}-\frac{v_{31} v_{32}}{E_{3}} & \text { plane strain }
\end{array},\right.
\end{aligned}
$$

and $u_{1}^{(k)}$ is the displacement component $u_{1}$ at the $k$-th node on the quarter-point isoparametric element; $E_{i}$ is the Young modulus in the principal $x_{i}$-direction and $v_{i j}$ is the Poisson`s ration defining the extensional strain in $\mathrm{x}_{\mathrm{j}}$-direction produced by a unit compressive strain in the $x_{i}$-direction. 
Alternatively the T-stress formula can be derived employing the nodal displacements perpendicular to the crack plane. Equating the coefficients of " $r$ " for the displacements $u_{2}$ in the asymptotic field (35) to that along 1-4 of the quarter point element (Fig.1) one can write

$$
\begin{gathered}
T=\frac{2}{a_{12} L}\left[u_{2}^{(1)}-2 u_{2}^{(8)}+u_{2}^{(4)}\right], \\
\text { where } a_{12}=\left\{\begin{array}{ll}
-\frac{v_{12}}{E_{1}} & \text { plane stress } \\
-\frac{v_{12}}{E_{1}}-\frac{v_{31} v_{32}}{E_{3}} & \text { plane strain }
\end{array} .\right.
\end{gathered}
$$

\section{Numerical results}

\subsection{Verification of the FEM model with orthotropic material}

In the first test example, the central crack with orthotropic material subjected to tension according to Banks-Sills et al. (2005) is analyzed (see.Fig.2). This problem was originally solved by Bowie and Freese (1972). The dimensions of the finite strip with central crack of length $a=0.5$, length of strip $\mathrm{h}=1$ and width of $\mathrm{b}=1$ has been taken and due to the symmetry only one quarter is modeled. The orthotropic material properties are the same as in BanksSills et al. (2005), Young moduli $E_{11}=5, E_{22}=10$, Poisson ratio $v_{12}=0.1$, and shear modulus $\mu=2.941$. The normalized SIF, $K_{I} / \sigma \sqrt{\pi a}$, computed by displacement correlation technique is 1.455 , corresponds exactly with the value given by Banks-Sills et al. (2005) (see Table.1). The discretization is performed by 1577 elements, quadratic 8-node elements and 34 singular triangular 6-nodes quarter point crack-tip elements. It appears that it is sufficiently fine discretization.
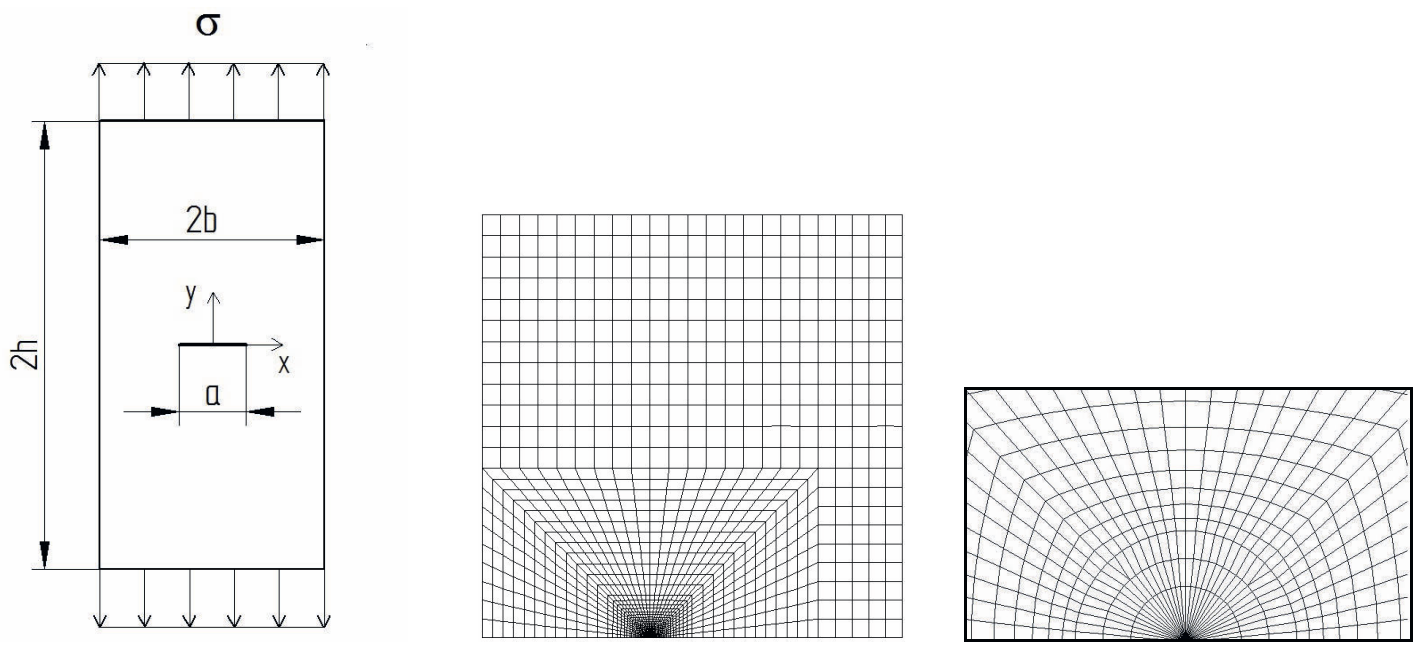

Fig. 2 Central crack in finite strip (left), FE-model (right) and detail of the mesh near the crack tip (right) 


\subsection{Verification of the FEM model with FGM isotropic material}

In the second test example the edge crack model (see.Fig.3) with FGM isotropic material properties according to the model in Kim and Paulino (2002) is analyzed. The same problem has been solved by Erdogan and Wu (1997) early. The finite strip with crack length $a=0.4$ and length $\mathrm{L}=8$ and width $\mathrm{W}=1$ is considered here. The applied load $\sigma=1$ is considered too. Material properties are assumed to be graded along the ${ }^{x}$-axis. The variation of Young modulus is $E=E_{1}^{\beta x}, \beta=\mathrm{E}_{2} / \mathrm{E}_{1}, \mathrm{E}_{1}=1$. The gradation of the material properties in ${ }^{x}$-direction enables to use symmetry conditions, therefore the displacement $u_{y}=0$ is prescribed on the bottom line ahead the crack tip. One half of the model is considered. The normalized SIF given by Kim and Paulino (2002) is 1.583 for the case of $E_{2} / E_{1}=10$. It is observed a very good agreement of Kim \& Paulino and present computational method results with relative error 0.31 percent. The comparison of the results is shown in a Table.1. The finite element model consists of 2451elements, quadratic 8-node elements and 34 triangular 6-nodes quarter point crack-tip elements.
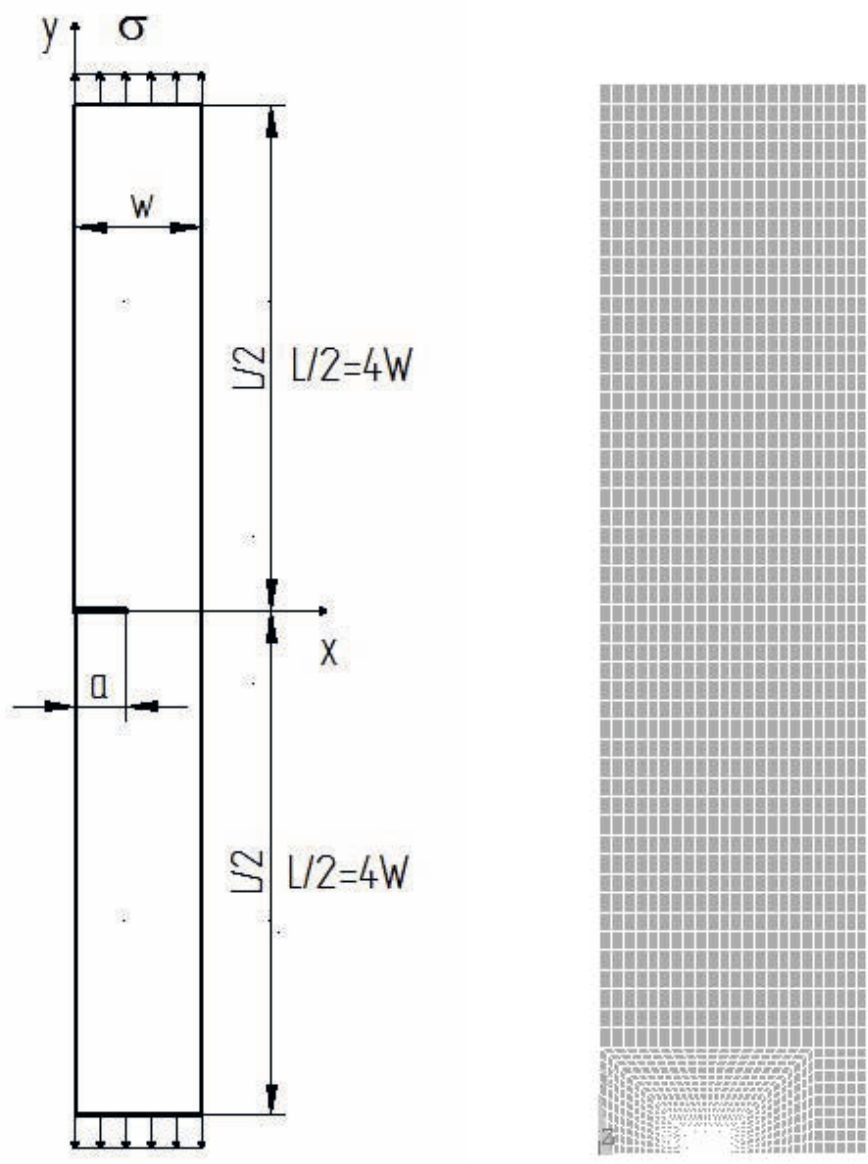

Fig. 3 Finite strip with edge crack (left), FE-model with symmetry (right) 
Table 1 The normalized stress intensity factors for orthotropic and FGM isotropic plates

\begin{tabular}{|c|c|c|}
\hline method & Orthotropic & FGM isotropic \\
\hline Kim and Paulino (2002) & - & 1.583 \\
\hline Banks-Sills et al. (2005) & 1.455 & - \\
\hline DCT (verification results) & 1.455 & 1.588 \\
\hline
\end{tabular}

\subsection{A hollow cylinder with two radial edge cracks in opposite directions}

In the third numerical example, an infinite hollow cylinder with two radial edge cracks in opposite directions is analyzed. The cracks are situated on internal surface of the hollow cylinder. A permanent temperature gradient is prescribed with temperature $\theta_{1}=30 \mathrm{deg}$ on the internal surface (radius $R_{1}=8 \mathrm{~cm}$ ) and $\theta_{2}=200 \mathrm{deg}$ on the external surface $\left(R_{2}=10 \mathrm{~cm}\right)$. Because of the symmetry, only a half of the cross section is analyzed with the boundary conditions shown in Fig.4. To test the computer code we have selected homogeneous isotropic material properties: Young modulus $E=7.8 \times 10^{4} \mathrm{MPa}$, Poisson ratio $v=0.3$ and thermal expansion coefficient $\alpha=0.125 \times 10^{-4} \mathrm{deg}^{-1}$. The crack length is denoted by $a$. The normalized stress intensity factor is defined as:

$$
f_{I}=\frac{K_{I}}{\sqrt{\pi a}} \frac{1-v}{\left(\theta_{2}-\theta_{1}\right) \alpha E}
$$

Variation of the normalized SIF with the crack length is presented in Fig. 5. One can observe that SIF increases with increasing the length of cracks.
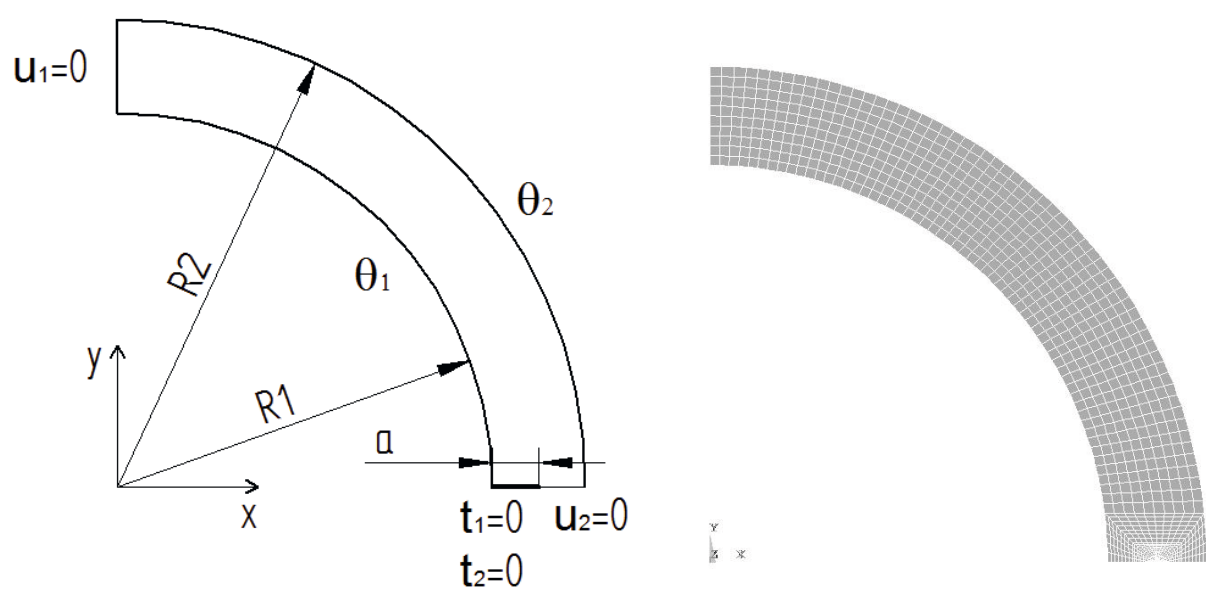
Fig. 4 Hollow cylinder with boundary conditions (left), FE-model (right)

The normalized T-stress is frequently called in literature as the biaxiality parameter is denoted as: $\quad B=\frac{T}{\sigma_{0}}=\frac{T \sqrt{\pi a}}{K_{I}}$.

The normalized T-stresses varies with crack length according the Fig.6.

In the next example, the same problem as previous has been analyzed with FGM orthotropic material properties. The exponential gradations of Young modulus and thermal expansion coefficient are considered along radial coordinate:

$$
E_{11}=E_{10} e^{\gamma r}, r=\sqrt{x^{2}+y^{2}}, \alpha=\alpha_{1} e^{\delta r},
$$

where $\gamma=0.25, \delta=0.1$ and following orthotropic material coefficients are considered: $E_{10}=7.8 \times 10^{4} M P a, E_{22}=2 E_{10}=15.6 \times 10^{4} M P a, v_{12}=0.3, \mu=E_{11} / 2(1+v)$.

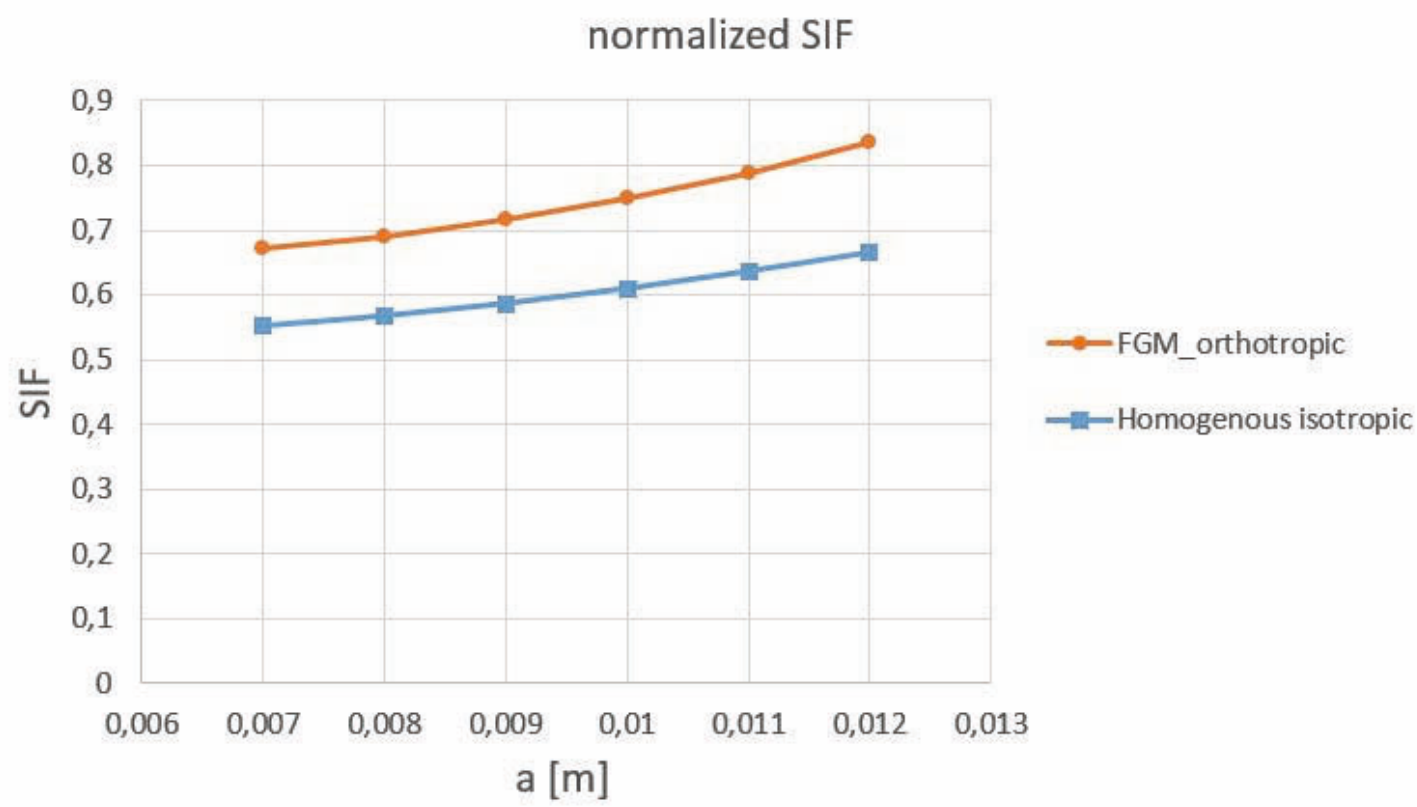

Fig. 5 Normalized stress intensity factor versus crack length a [m] 


\section{T-stress vs crack length}

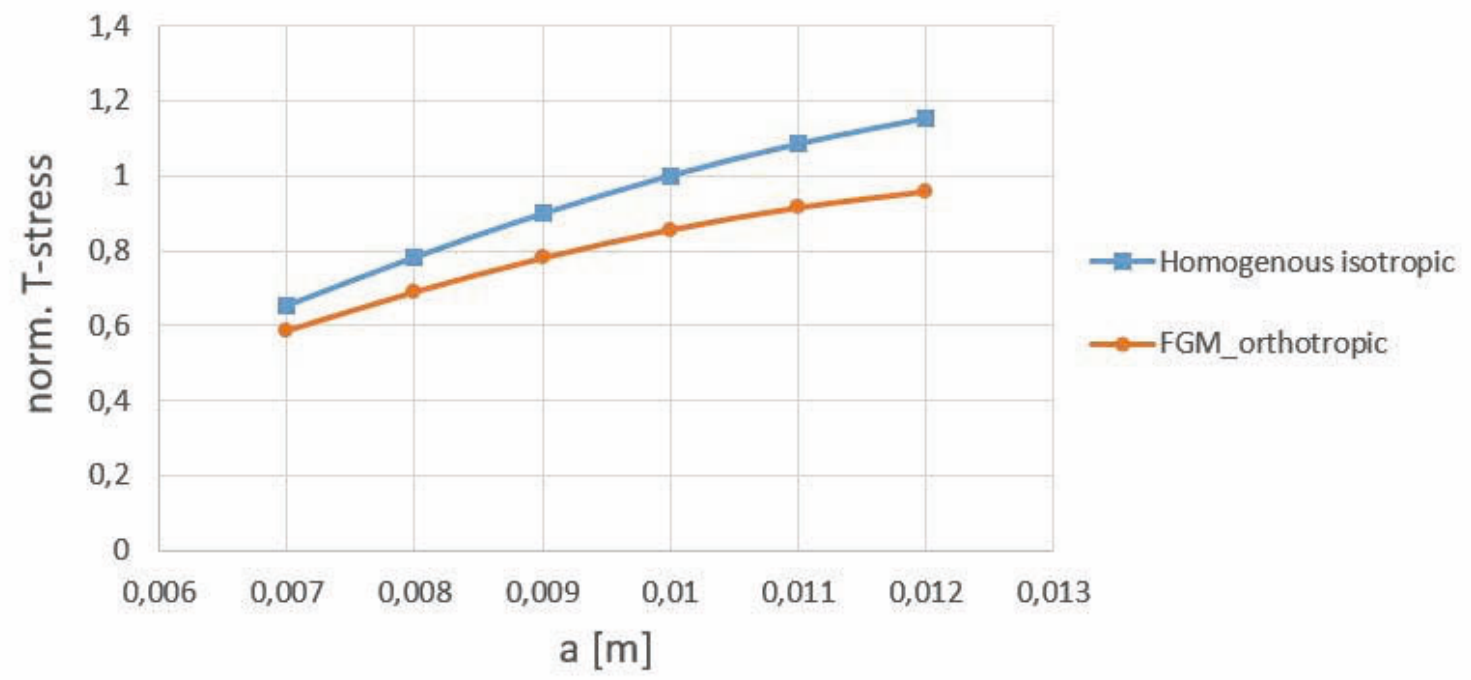

Fig. 6 Normalized T-stress versus crack length $a[\mathrm{~m}]$

The numerical results for variation of the normalized SIF and the T-stress with the crack length are presented in Figs. 5 and 6, respectively.

\section{Conclusions}

This paper presents computational methods for the evaluation of stress intensity factors and $\mathrm{T}$-stresses for crack problems in orthotropic functionally graded material bodies under thermal loads. The FEM formulation for solution of related initial-boundary value problem within coupled theory of thermoelasticity has been developed for orthotropic materials with functionally graded material coefficients. Making use of asymptotic expansions for displacements and stresses in the near vicinity of the crack tip and using the quarter-point crack-tip elements, we obtained the formulae for direct evaluation of the SIFs and T-stress factor directly from the nodal values of displacements on the crack-tip elements (displacement correlation technique - DCT). In order to verify the derived formulae, we considered several test examples from the elastostatics for which the benchmark solutions are available. Perfect agreement with literature results for the SIFs has been obtained in homogeneous orthotropic finite strip with central crack as well as in FGM orthotropic finite strip with edge crack. The dependence of the SIF and the T-stress term on the length of the crack has been studied numerically for two edge cracks symmetrically distributed in an infinite hollow cylinder subject to stationary thermal loading with prescribed temperatures on the interior and exterior surfaces. The material properties have been considered either homogeneous isotropic or orthotropic with functionally graded coefficients. The proposed technique proved to be reliable and it will be employed in future also in numerical analyses of transient problems.

\section{Acknowledgements}

Volume 65, No.1, (2015) @ @2015 SjF STU Bratislava 
The first three authors acknowledge the support of the Slovak Science and Technology Assistance Agency registered under number APVV-14-0216.

\section{REFERENCES}

[1] Aoki, S.; Kishimoto, K. and Sakata, M. (1981) Crack tip stress and strain singularity in thermally loaded elastic-plastic materials. J. Appl. Mech., 48, 428-429.

[2] Barsoum, R.S. (1976) On the use of isoparametric finite elements in linear fracture mechanics. Int. J. Num. Meth. Engn. 10, 25-37.

[3] Betegon, C. and Hancock, J.W. (1991) Two parameter characterization of elastic-plastic crack tip fields. J. Appl. Mech., 58, 104-110.

[4] Cotterell, B. and Rice, J.P. (1980) Slightly curved or kinked cracks. Int. J. Fracture, 16, 155-169.

[5] Dolbow, J. and Gosz, M. (2002) On the computation of mixed-mode stress intensity factors in functionally graded materials. Int. J. Solids Structures, 39, 2557-2574.

[6] Eischen, J.W. (1987) Fracture of nonhomogeneous materials. Int. J. Fracture 34, 3-22.

[7] Erdogan, F. (1995) Fracture mechanics of functionally graded materials. Compos. Eng., 5, 753770.

[8] Eshelby, J.D; Read, W.T. and Shockley, W. (1953) Anisotropic elasticity with applications to dislocations. Acta Metallurgica, 1, 251-259.

[9] Gao, H. and Chiu, C.H. (1992) Slightly curved or kinked cracks in anisotropic elastic solids. Int. J. Solids Structures, 29, 947-972.

[10] Gu, P. and Asaro, R.J. (1997) Cracks in functionally graded materials. Int. J. Solids and Structures, 34, 1-17.

[11] Gupta, M., Alderliesten, R.C., Benedictus, R. (2015) A review of T-stress and its effects in fracture mechanics. Engng Fract Mech 134,218-241.

[12] Jin, Z.H. and Noda, N. (1993a) Minimization of thermal stress intensity factor for a crack in a metal-ceramic mixture. Trans. American Ceramic Soc. Functionally Gradient Materials, 34, 4754.

[13] Jin, Z.H. and Noda, N. (1993b) An internal crack parallel to the boundary of a nonhomogeneous half plane under thermal loading. Int. J. Engn. Sci., 31, 793-806.

[14] Jin, Z.H. and Noda, N. (1994) Crack tip singular fields in nonhomogeneous materials. J. Appl. Mech., 64, 738-739.

[15] Kfouri, A.P. (1986) Some evaluations of the elastic T-term using Eshelby's method. Int. J. Fracture, 30, 301-315.

[16] Kim, J.H. and Paulino, G.H. (2002) Finite element evaluation of mixed mode stress intensity factors in functionally graded materials. Int. J. Num. Meth. Engn., 53, 1903-1935.

[17] Kim, J.H. and Paulino, G.H. (2003a) T-stress, mixed mode stress intensity factors, and crack initiation angles in functionally graded materials: A unified approach using the interaction integral method. Computer Meth. Appl. Mech. Engn., 192, 1463-1494.

[18] Kim, J.H. and Paulino, G.H. (2003b) The interaction integral for fracture of orthotropic functionally graded materials: Evaluation of stress intensity factors. Int. J. Solids and Structures, 40, 3967-4001

[19] Kim, J.H. and Paulino, G.H. (2004) T-stress in orthotropic functionally graded materials: Lekhnitskii and Stroh formalisms. Int. J. Fracture, 126, 345-384.

[20] Kishimoto, K.; Aoki, S. and Sakata, M. (1980) On the path independent integral - $\hat{J}$. J. Engn. Fracture Mech., 13, 841-850.

[21] Larsson, S.G. and Carlsson, A.J. (1973) Influence of nonsingular stress terms and specimen geometry on small-scale yielding at crack tips in elastic-plastic materials. J. Mech. Phys. Solids, 21, 263-277.

[22] Lekhnitskii, S.G. (1963) Theory of Elasticity of an Anisotropic Body. Holden Day.

[23] Leevers, P.S. and Radon, J.C. (1982) Inherent stress biaxiality in various fracture specimen geometries. Int. J. Fracture, 19, 311-325. 
[24] Nakamura, T. and Parks, D.M. (1982) Determination of elastic T-stresses along three-dimensional crack fronts using an interaction integral. Int. J. Solids Structures, 29, 1597-1611.

[25] Nemat-Alla, M. and Noda, N. (1996) Thermal stress intensity factor for functionally gradient half space with an edge crack under thermal load. Archive Appl. Mech., 66, 569-580.

[26] Noda, N. and Jin, Z.H. (1993a) Thermal stress intensity factors for a crack in a functionally gradient material. Int. J. Solids Structures, 30, 1039-1056.

[27] Noda, N. and Jin, Z.H. (1993b) Steady thermal stresses in an infinite nonhomogeneous elastic solid containing a crack. J. Thermal Stresses, 16, 181-196.

[28] Noda, N. and Jin, Z.H. (1995) Crack tip singularity fields in non-homogeneous body under thermal stress fields. JSME Int. Jour. Ser. A, 38, 364-369.

[29] Nowacki, W. (1986), Thermoelasticity, Pergamon, Oxford.

[30] Olsen, P.C. (1994) Determining the stress intensity factors $K_{I}, K_{I I}$ and the T-term via the conservation laws using the boundary element method. Engn. Fracture Mech., 49, 49-60.

[31] Ozturk, M. and Erdogan, F. (1997) Mode I crack problem in an inhomogeneous orthotropic medium. Int. J. Engn. Sci., 35, 869-883.

[32] Ozturk, M. and Erdogan, F. (1999) The mixed mode crack problem in an inhomogeneous orthotropic medium. Int. J. Fracture, 98, 243-261.

[33] Phan, A.V. (2011) A non-singular boundary integral formula for determining the T-stress for cracks of arbitrary geometry. Eng. Fracture Mech. 78, 2273-2285.

[34] Paulino, G.H.; Jin, Z.H. and Dodds, R.H. (2003) Failure of functionally graded materials. In: Karihaloo B, Knauss WG, editors, Comprehensive Structural Integrity, Volume 2, Elsevier Science, 607-644.

[35] Paulino, G.H. and Kim, J.H. (2004) A new approach to compute T-stress in functionally graded materials by means the interaction integral method. Engn. Fracture Mechanics, 71, 1907-1950.

[36] Rao, B.N. and Rahman, S. (2003) Mesh-free analysis of cracks in isotropic functionally graded materials. Engn. Fracture Mechanics, 70, 1-27.

[37] Rice JR. (1974) Limitations to the small scale yielding approximation for crack tip plasticity. J Mech Phys Solids 22, 17-26.

[38] Selvarathinam A.S., Goree J.G. (1998) T-stress based fracture model for cracks in isotropic materials. Engng Fract Mech 60,543-61.

[39] Shah, P.D.; Tan, C.L. and Wang, X. (2006) T-stress solutions for two-dimensional crack problems in anisotropic elasticity using the boundary element method. Fatigue Fract. Engn. Mater. Struc. 29, 343-356.

[40] Sherry, A.H.; France, C.C. and Goldthorpe, M.R. (1995) Compendium of T-stress solutions for two and three dimensional cracked geometries. Fatigue Fract. Engn. Mater. Struct., 18, 141-155.

[41] Sih, G.C.; Paris, P.C. and Irwin, G.R. (1965) On cracks in rectilinearly anisotropic bodies. Int. J. Fracture Mechanics, 1, 189-203

[42] Sladek, J. and Sladek, V. (1997a) Evaluations of the T-stress for interface cracks by the boundary element method. Engn. Fracture Mech., 56, 813-825.

[43] Sladek, J. and Sladek, V. (1997b) Evaluation of T-stresses and stress intensity factors in stationary thermoelasticity by the conservation integral method. Int. J. Fracture, 86, 199-219.

[44] Sladek, J.; Sladek, V. and Fedelinski, P. (1997c) Integral formulation for elastodynamic Tstresses. Int. J. Fracture, 84, 103-116.

[45] Sladek, J.; Sladek, V. and Zhang, Ch. (2005) An advanced numerical method for computing elastodynamic fracture parameters in functionally graded materials. Computational Materials Science, 32, 532-543.

[46] Sladek, J., Sladek, V., Zhang, Ch., Tan, C.L. (2006) Evaluation of fracture parameters for crack problems in FGM by a meshless method. Journal Theoretical and Applied Mechanics, 44, 603636.

Volume 65, No.1, (2015) @ @2015 SjF STU Bratislava 
[47] Smith, D.J.; Ayatollahi, M.R. and Pavier, M.J. (2001) The role of T-stress in brittle fracture for linear elastic materials under mixed-mode loading. Fatigue Fracture Engn. Material Structure, 24, 137-150.

[48] Sumpter, J.D.G. (1993) An experimental investigation of the T-stress approach, in: Constraint Effects in Fracture, ASTM STP 1171, 429-508.

[49] Sumpter, J.D.G. and Hancock, J.W. (1991) Shallow crack toughness of HY80 welds - an analysis based on T. Int. J. Press. Vess. Piping, 45, 207-229.

[50] Suresh, S. and Mortensen, A. (1998) Fundamentals of Functionally Graded Materials. Institute of Materials, London.

[51] Tan, C.L., Wang, X. (2003) The use ofquarter-point crack-tip elements for T-stress determination in boundary element method analysis. Eng. Fracture Mech. 70, 2247-2252.

[52] Yue, Z.Q.; Xiao, H.T. and Tham, L.G. (2003) Boundary element analysis of crack problems in functionally graded materials. Int. J. Solids and Structures, 40, 3273-3291.

[53] Williams, M.L. (1957) On the stress distribution at the base of stationary crack. J. Appl. Mech., $24,109-114$.

[54] Williams, J.G. and Ewing, P.D. (1972) Fracture under complex stress - the angled crack problem. J. Fracture Mech., 8, 441-446. 